\title{
Specifying social cognitive processes with a social dual-task paradigm
}

\section{Roman Liepelt ${ }^{*}$, Anna Stenzel and Markus Lappe}

Institute for Psychology, University of Muenster, Muenster, Germany

\section{Edited by:}

Bert Timmermans, University

Hospital Cologne, Germany

\section{Reviewed by:}

Nancy Zucker, Duke University

Medical Center, USA

Lorenza S. Colzato, Leiden

University, Netherlands

\section{*Correspondence:}

Roman Liepelt, Institute for

Psychology, University of Muenster,

48149 Munster, Germany.

e-mail: roman.liepelt@uni-

muenster.de
Automatic imitation tasks measuring motor priming effects showed that we directly map observed actions of other agents onto our own motor repertoire (direct matching). A recent joint action study using a social dual-task paradigm provided evidence for task monitoring. In the present study, we aimed to test (a) if automatic imitation is disturbed during joint action and (b) if task monitoring is content or time dependent. We used a social dual-task that was made of an automatic imitation task (Person 1: Task 1) and a two-choice number task (Person 2: Task 2). Each participant performed one of the two tasks, which were given with a variable stimulus onset asynchrony (SOA), in an individual and a joint condition. We found a regular motor priming effect in individual and joint conditions. Under joint conditions, we replicated the previous finding of an increase of reaction times for Person 2 with decreasing SOA. The latter effect was not related to the specific responses performed by both persons. Further, we did not find evidence for a representation of the other's specific S-R mappings. Our findings suggest that (a) automatic imitation is not disturbed during joint action and (b) task monitoring is time dependent.

Keywords: joint action, social cognition, social PRP, dual-task

\section{INTRODUCTION}

When somebody performs two tasks at the same time performance either in one (Pashler and Johnston, 1989) or both of the tasks (Tombu and Jolicoeur, 2004) is reduced, because the cognitive system has only a limited amount of capacity for information processing. When Task 1 is prioritized, reaction times in Task 2 increase with decreasing Stimulus Onset Asynchrony (SOA) between the tasks (Telford, 1931; Welford, 1952). This is known as the psychological refractory period (PRP). The PRP effect is often explained as a capacity limit either by an inherent structural (Pashler, 1984, 1994) or a voluntary strategic (Meyer and Kieras, 1997a; Schumacher et al., 2001) bottleneck. More recent approaches also discuss the role of crosstalk between the two responses, linking the PRP to spatially mediated responseresponse (R-R) compatibility effects (e.g., Hommel, 1998; Lien and Proctor, 2000; Schubert et al., 2008; Liepelt et al., 2011a). This response-response crosstalk helps to reduce dual-task costs under compatible R-R conditions (e.g., left-left), but hinders dual-task cost reduction under incompatible conditions (e.g., left-right).

When two individuals share a task the performance of one sometimes depends on the actions of the other. This has been shown in studies on multi person action coordination. This research originated from experiments on action observation. Action observation activates brain areas that are involved in motor preparation and execution of one's own action (Gallese et al., 1996; Iacoboni et al., 1999; Buccino et al., 2001; Fogassi et al., 2005). Behavioral and neuroscientific work on automatic imitation (Brass et al., 2000; Liepelt et al., 2008a) and action understanding (Iacoboni et al., 2005; Liepelt et al., 2008b) suggests that action observation automatically activates an internal action simulation process (Gallese and Goldman, 1998; Graf et al.,
2007) in the observer that can be used to predict others actions (Springer et al., 2011; Stadler et al., 2011) and to understand their goal states (Liepelt et al., 2010). Taken together, this leads to the assumption that people directly map observed actions of other agents onto their own motor repertoire (direct matching hypothesis, Iacoboni et al., 1999). Direct matching can be studied with an automatic imitation paradigm (Brass and Heyes, 2005) in which participants carry out simple finger-lifting movements while observing congruent or incongruent finger movements on the screen. For example, if participants have to lift their finger in response to a symbolic number cue ("1" or "2") while observing a task-irrelevant finger-lifting movement of another person their action is facilitated if the movement is congruent and slowed-down if it is incongruent (Brass et al., 2000; Bertenthal et al., 2006). The difference in reaction times between congruent and incongruent conditions is termed motor priming effect (Liepelt and Brass, 2010a,b). Here social cognitive processes are investigated in a single individual observing another person's action. In contrast, joint action has been defined as any form of social interaction where two or more individuals coordinate their actions in space and time to bring about a change in the environment (Sebanz et al., 2006). Joint action requires a close match of externally and internally triggered action representations, which are also supposed to be mediated by internal real-time simulation (Sebanz and Knoblich, 2009), action prediction (Schubotz, 2007; Springer et al., 2011) and a fine tuned action coordination process aligning dyadic actions in space and time (Sebanz et al., 2005, 2006).

When two individuals share two tasks, the combination and distribution of the tasks may induce processing bottlenecks between the two subjects. Indeed, joint action coordination in a social version of the dual-task paradigm (Liepelt and Prinz, 
2011) induces bottleneck-like processing when two individuals share two complex and relatively independent number magnitude tasks. Participants had to indicate whether two numbers (1-4 and 6-9) appearing one above the other were smaller or larger than five. As in the standard PRP research, number stimuli were presented with a variable SOA so that the upper number (Stimulus for Person 1 sitting on the left side) mostly appeared earlier in time than the lower number (Stimulus for Person 2 sitting on the right side). The dual-task was distributed between two people so that each person responded to only one of the numbers. When the instructions prioritized Task 1 processing reaction times of Person 2 (Task 2) mimicked the standard PRP effect typically observed when one person has to perform both tasks simultaneously. Because reaction times for Person 2 also increased with decreasing SOA (social PRP effect), Liepelt and Prinz (2011) interpreted this finding as evidence for a strategic task monitoring effect that is related to the given task requirements (Meyer and Kieras, 1997b).

In the present manuscript, we investigate two central questions that originate from this research. First, we ask whether direct matching occurs when two persons are engaged in joint action or whether joint action poses additional demands on our cognitive system so that direct matching is disturbed. The former should be expected, when direct matching previously found in automatic imitation research (Brass et al., 2000; Liepelt et al., 2010) is also present during joint action (Sebanz et al., 2003; Sebanz and Knoblich, 2009). The second question concerns the exact nature of the task monitoring effect in the social dual-task paradigm. We ask whether task monitoring is task-content specific (i.e., specific to the action produced by both co-actors or specific to the $\mathrm{S}-\mathrm{R}$ mappings of both co-actors), or whether task monitoring is related to the time at which the action effect is produced.

Aiming to answer these two questions, we combined an automatic imitation paradigm (Brass et al., 2000) and a social dualtask paradigm (Liepelt and Prinz, 2011). For the dual-task, we used an automatic imitation task as Task 1 and a two-choice number task as Task 2. We presented stimuli in two dimensions: For the automatic imitation task, we presented a human hand stimulus (Brass et al., 2000) depicting a finger-lifting movement of either the left index or middle finger (Stimulus 1, S1) shown from a third person perspective. For the two-choice number task, we presented a number stimulus (digit 1 or 2; Stimulus 2, S2) presented between the two moving fingers of the hand stimulus (Figure 1A,B). S1 and S2 were given with a variable SOA. Each participant was responsible for one part of the dualtask, only. Person 1 performed index or middle finger-lifting movements (Response 1, R1) in response to the moving hand stimulus either in an imitative or counter-imitative way (Task 1). Person 2 responded to the number stimulus by lifting the index finger when the number 1 appeared, and the middle finger when number 2 appeared (Response 2, R2) representing Task 2 (Figure 1A,B). Participants performed their part of the dualtask alone (individual condition, Figure 1B) and together with a partner who took over the other part of the dual-task (joint condition, Figure 1A). In the joint condition priority was given on Task 1 and task order was explicitly instructed. Both participants responded with finger-lifting movements of their right hand.
For the present social dual-task situation, we had the following predictions for automatic imitation and output monitoring. If both persons (Person 1 and Person 2) directly match the fingerlifting action on the screen (representing the stimulus for Task 1) on their own action repertoire, one would predict a motor priming effect for Person 1 (Task 1), as well as a motor priming effect for Person 2 (Task 2). These motor priming effects should be present in the individual and in the joint condition, if joint-task processing does not disturb direct matching. In both conditions, this effect is expected at the shortest SOA condition where the observation-execution overlap is strongest.

Output monitoring assumes that the second person acting on the second task monitors the action effects of the first person's task that commences earlier in time. If the social PRP effect (Liepelt and Prinz, 2011) is due to strategic action effect monitoring, one would predict an increase of reaction times for Task 2 (Person 2) in the joint condition when using a fixed task order and a priority instruction on Task 1 processing. This effect should be larger than in the individual condition where no action effect monitoring is supposed to take place. If action effect monitoring takes place, one could also expect a complete transfer of the S1-R1 compatibility effect (motor priming effect in Task 1, Person 1) to the reaction times of Task 2 (Person 2) at the zero SOA condition.

This action effect monitoring may either be content specific or time dependent. If both persons do not only supervise when the other person responds (time dependent action effect monitoring), but also which specific action the other person performs (content specific action effect monitoring), one would predict that the second person responds faster in the joint condition of Task 2 when activating the spatially (and anatomically) identical finger response as the first person in Task 1 (e.g., index-index), who acts ahead in time, as when both persons activate spatially (and anatomically) different finger responses (e.g., index-middle). We will use the term response-response crosstalk (Schubert et al., 2008) for the latter kind of relation. This effect would reflect the content of the specific responses given by the other person. Content specificity may, however, also refer to more than just the simple action or action effect produced by the other person. The content may also include the specific task rule for the other person, i.e., with which S-R mappings the other person responds. A monitoring of the exact task rule may predict that the S1-R2 compatibility effect (the motor priming effect for Person 2) is larger when Person 1 responds imitatively, as when he responds counter-imitatively to the hand stimulus. However, if action effect monitoring is time dependent (and not content dependent), one would not predict a speed up of reaction times in Task 2 (Person 2) of the joint condition when both persons sharing the dual-task activate the same finger responses and no effect of task rule of the other person on the motor priming effect of Person 2.

\section{METHOD \\ PARTICIPANTS}

Thirty-two participants took part in the experiment (Mean age $=$ 26.3 years, $\mathrm{SD}=6.0$ years; 19 female; 29 right-handed). All of them participated in the individual condition as well as in the joint condition. Half of the participants were randomly assigned 


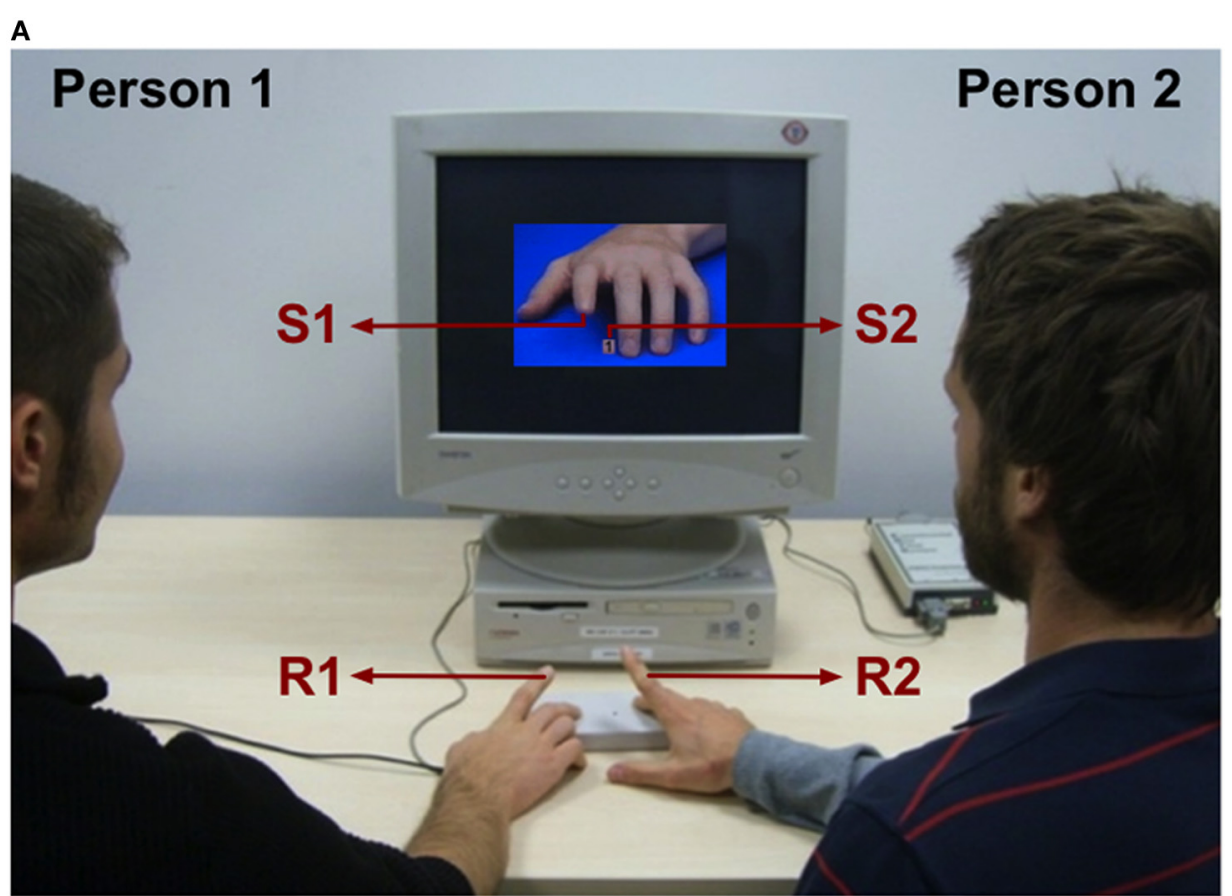

B

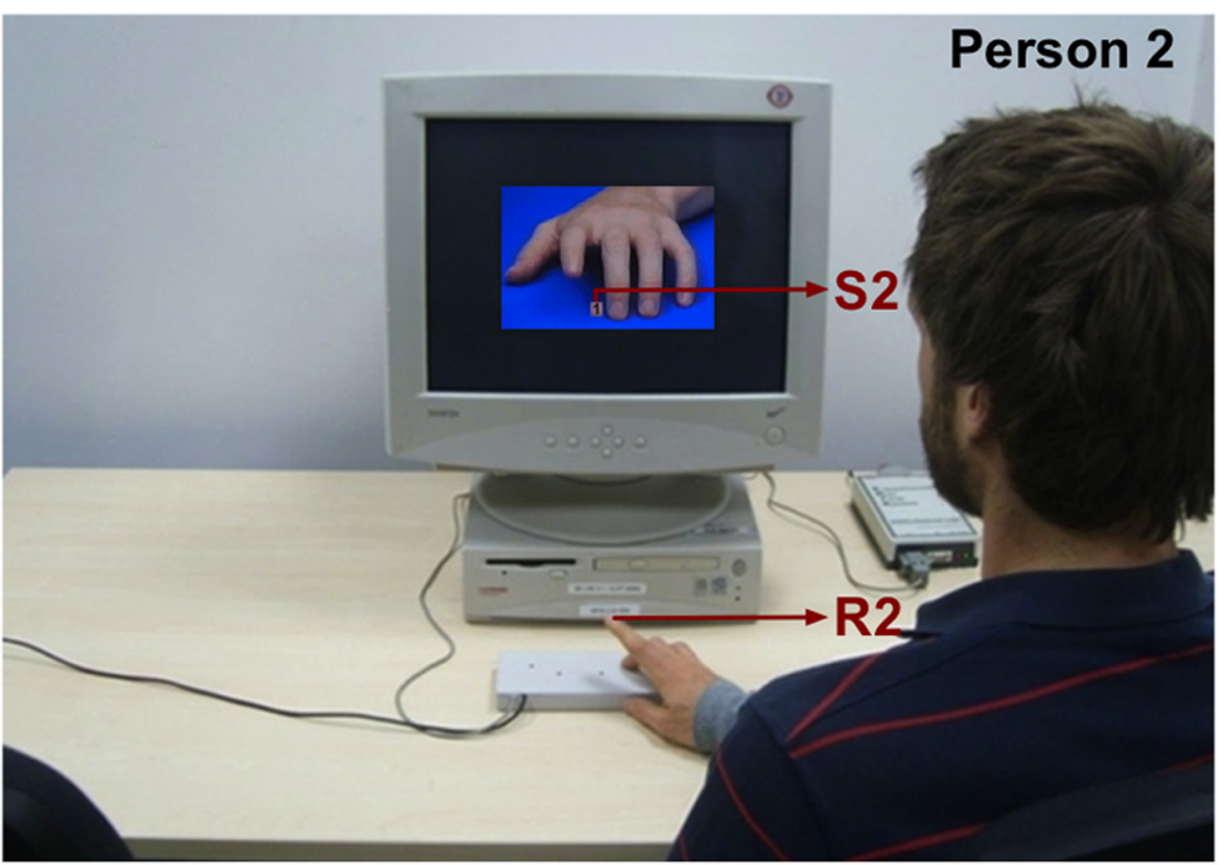

FIGURE 1 | Experimental setup. Each participant performed one part of a dual-task. Person 1 (acting on Task 1) sitting on the left side had to execute finger-lifting movements (R1) with his right index or middle finger in response to a hand stimulus (S1). Person 2 (acting on Task 2) sitting on the right side had to respond with his right index or middle finger (R2) in response to a number stimulus (S2). (A) Both individuals perform their part of the dual-task together (joint condition). (B) One individual (Person 2) performs his part of the dual-task alone (individual condition). to Task 1 and the other half to Task 2. All participants had normal or corrected-to-normal vision, and were naive with regard to the hypotheses of the experiment. Each participant was paid $€ 7$ or student credit points for participation. Participants gave their informed consent to participate. The experiment was conducted in accordance with the standards of the ethics committee of the University of Münster and the ethical standards laid down in the 1975 Declaration of Helsinki. 


\section{APPARATUS AND STIMULI}

The experiment was conducted in a sound-attenuated and dimly lit room. The stimuli were displayed on a 17 -in color monitor that was connected to a Pentium I PC. For stimulus presentation we used ERTS software (Experimental Runtime System; Beringer, 2000).

As stimuli, we used short sequences of finger movements and number stimuli (digit 1 or 2). The sequence of finger movements (adapted from Brass et al., 2000) consisted of two frames producing the impression of a finger-lifting movement showing a hand from a third person perspective. The hand was presented at the center of the monitor. The first frame showed a left human hand in a resting position. The second frame showed the same hand with either a lifted index or middle finger. As all participants responded with their right hand, the hand on the screen mirrored both participants' response hands. The number stimulus, a black digit (1 or 2) displayed on a light-colored squared background, appeared between the index and middle finger of the hand stimulus. At a viewing distance of $80 \mathrm{~cm}$, the animated hand subtended a visual angle of approximately $11.8^{\circ} \times$ $7.4^{\circ}$. The number stimulus had a visual angle of approximately $0.64^{\circ} \times 0.72^{\circ}$.

Reaction times (RTs) were recorded with an optical response device, which detected the initiation of the finger-lifting movement of both participants. The device was equipped with four optical sensors, which participants covered with the index and middle finger of their right hands.

\section{PROCEDURE AND DESIGN}

Each participant was responsible for one part of the dual-task, only. Participants performed their part of the dual-task alone (individual condition, Figure 1B) and together with a partner who took over the other part of the dual-task (joint condition, Figure 1A). We used a within-subjects design, i.e., all subjects participated in the joint and in the individual condition. The order of conditions was counterbalanced across participants (Figure 2).

Person 1 responded to the observed finger movement (Task 1). Person 2 responded to the number stimulus (Task 2). Both participants reacted by lifting either the index finger or the middle finger of their right hand. Each condition was split into two blocks. In one of the two blocks, Person 1 responded in an imitative (S1-R1 compatible) way to the observed finger movement (i.e., lifting the index finger, when the observed hand lifted the index finger; lifting the middle finger, when the hand lifted the middle finger). In the other block, Person 1 responded in a counter-imitative ( $\mathrm{S} 1-\mathrm{R} 1$ incompatible) way to the observed finger movement (Figure 2). The S1-R1 compatibility indicating the relationship between the perceived hand stimulus (S1) and the executed response finger of Person 1 (R1) was kept constant within one block and varied block wise. In half of the pairs, the respective Person 1 started with the imitative block, in the other half of pairs, Person 1 started with the counterimitative block, so that the order of blocks was counterbalanced across participants. In contrast, the task of Person 2 was the same throughout the entire experiment. In both blocks of each condition (joint and individual condition), Person 2 responded

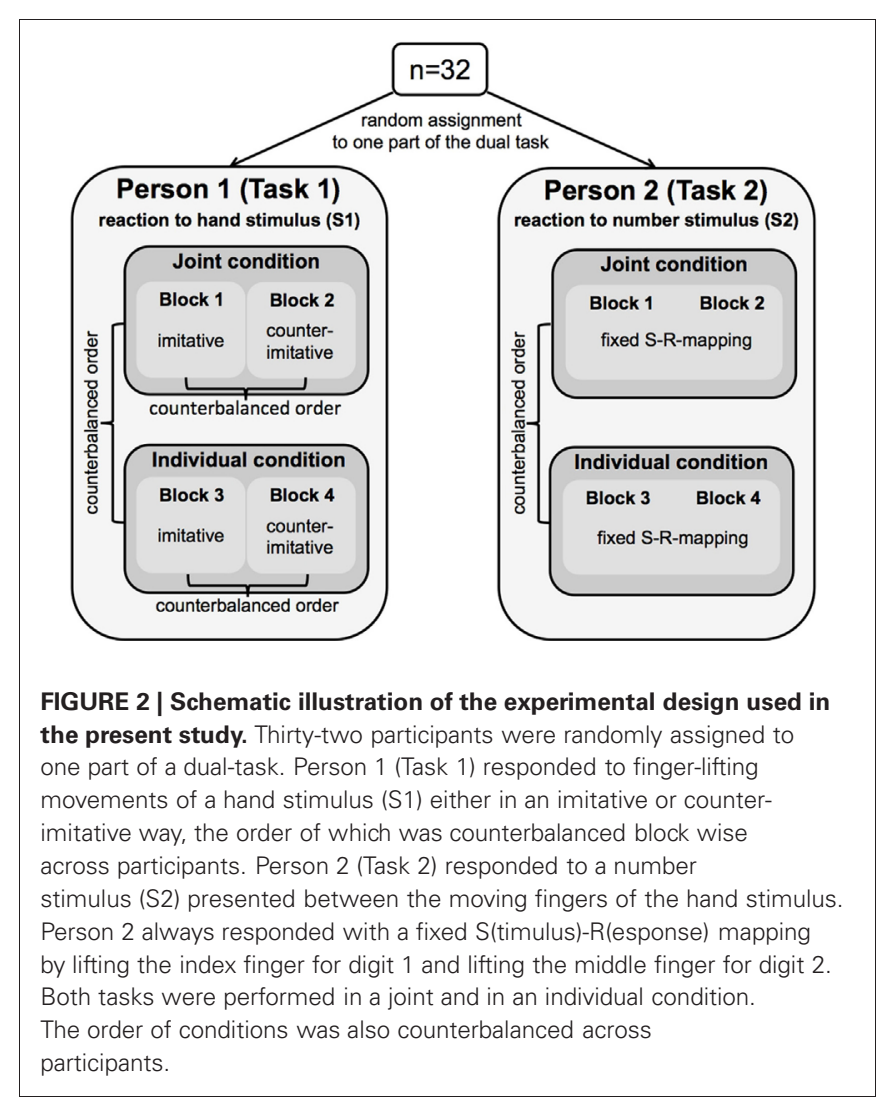

with a fixed S-R mapping by lifting the index finger when the digit 1 appeared on the screen, and by lifting the middle finger when the digit 2 appeared (Figure 2). S1-R2 compatibility for Person 2 (i.e., the compatibility between the hand stimulus, S1, and the response of Person 2, R2) varied within each block, and was based on the combination of the presented number stimulus and the displayed finger-lifting response, respectively. This could result either in an automatic imitative response (S1-R2 compatible trial) or a counter-imitative response (S1-R2 incompatible trial).

In the joint condition participants were instructed to give priority to Task 1 (Pashler, 1994; Liepelt and Prinz, 2011) inducing a specific response order. We explicitly instructed Person 2 to wait with the response to the number stimulus until the response of Person 1 was completed. This was done to specifically test for output monitoring as a possible basis of the social PRP effect observed in previous studies. Person 1 was instructed to respond directly after the finger-lifting movement was displayed on the screen. In all conditions, all participants were encouraged to respond as quickly and accurately as possible.

For each participant the sitting position was kept constant in both conditions: Person 1 performing Task 1 was always seated on the left side of the monitor, whereas Person 2 performing Task 2 was always seated on the right side. In the joint condition, participants were seated next to each other, in the individual condition an empty chair remained beside the participant.

Each trial began with the presentation of the resting hand for $800 \mathrm{~ms}$. Afterwards the hand lifted either the index or middle 
finger for $1400 \mathrm{~ms}$. With a variable SOA of $0,100,300$, or $1000 \mathrm{~ms}$ after finger movement onset, the number stimulus (digit 1 or 2) appeared and stayed on the screen together with the hand stimulus for $1400 \mathrm{~ms}$. Following correct responses, the screen remained blank. If no response was given in this time interval, the feedback "zu langsam" (too slow) was presented. In the case of an incorrect response, error feedback "Fehler" (error) was provided. All feedbacks (blank, too slow, or error) were displayed for $300 \mathrm{~ms}$. After the feedback, a constant inter-trial interval was given for $2100 \mathrm{~ms}$. The total trial duration amounted to $4600 \mathrm{~ms}$ plus SOA. To control for perceptual differences between conditions, we used the same stimuli in individual and joint conditions.

The joint condition and the individual condition consisted of 256 trials each. Both conditions were split into two blocks of 128 trials (an imitative block and a counter-imitative block). Participants had a short break between blocks and within each block (after 64 trials). The order of trials was randomized within each block. At the beginning of each condition (individual and joint) participants were given 16 practice trials.

\section{RESULTS}

As a dependent measure RTs were analyzed using an analysis of variance (ANOVA) with different factors specified below. Errors (individual condition: Task 1: 1.9\%, Task 2: 2.3\%; joint condition: Task 1: $2.7 \%$, Task 2: $3.4 \%$ ) or RTs shorter than $150 \mathrm{~ms}$ or longer than $1800 \mathrm{~ms}$ (individual condition: Task 1: $0.02 \%$, Task 2: 0.15\%; joint condition: Task 1: 0\%, Task 2: 0\%) were excluded from reaction time analyses. A Greenhouse-Geisser correction was used to assess the significance of each effect for all analyses.

\section{MOTOR PRIMING EFFECTS}

To analyze motor priming effects for Person 2 and Person 1, we calculated a Two-Way ANOVA for the $0 \mathrm{~ms}$ SOA, separately for Task 2 and Task 1. The ANOVA included the within-subject factors condition (joint, individual) and compatibility (compatible, incompatible) between the hand stimulus (S1) and each subject's response (R2 or R1).

\section{Motor priming effect for task 2}

A significant main effect of condition, $F_{(1,15)}=67.94, p<$ 0.001 , partial $\eta^{2}=0.82$, indicated slower overall RTs in the joint condition ( $659 \mathrm{~ms}$ ) than in the individual condition (422 ms). A significant main effect of S1-R2 compatibility, $F_{(1,15)}=14.48$, $p<0.01$, partial $\eta^{2}=0.49$, indicated faster RTs for S1-R2 compatible trials $(529 \mathrm{~ms})$, as compared to S1-R2 incompatible trials (552 $\mathrm{ms}$ ) confirming the presence of a motor priming effect for Task 2. The magnitude of motor priming did not differ statistically between the joint $(18 \mathrm{~ms})$ and the individual condition ( $27 \mathrm{~ms})$, as indicated by a non-significant interaction between condition and S1-R2 compatibility, $F<1$, partial $\eta^{2}=0.06$.

\section{Motor priming effect for task 1}

A significant main effect of condition, $F_{(1,15)}=6.08, p<0.05$, partial $\eta^{2}=0.29$, indicated faster overall RTs in the joint condition $(357 \mathrm{~ms})$ than in the individual condition $(376 \mathrm{~ms})$. A significant main effect of S1-R1 compatibility, $F_{(1,15)}=57.96$, $p<0.001$, partial $\eta^{2}=0.79$, indicated faster RTs for S1-R1 compatible trials ( $327 \mathrm{~ms}$ ), as compared to S1-R1 incompatible trials
(407 ms) confirming the presence of a motor priming effect in Task 1. The magnitude of motor priming did not differ statistically between the joint $(75 \mathrm{~ms})$ and the individual condition $(86 \mathrm{~ms})$, as indicated by a non-significant interaction between condition and S1-R1 compatibility, $F<1$, partial $\eta^{2}=0.06$.

\section{TESTING FOR ACTION EFFECT MONITORING Social PRP effect}

To investigate if there is a social PRP effect, we calculated an ANOVA including the factors condition (joint, individual) and SOA $(0,100,300,1000 \mathrm{~ms})$, both as within-subjects variables, separately for Task 2 and Task 1.

Reaction time analysis for task 2. For Task 2, we found a significant main effect of condition, $F_{(1,15)}=57.24, p<0.001$, partial $\eta^{2}=0.79$, indicating slower overall RTs in the joint condition ( $539 \mathrm{~ms})$ as compared to the individual condition $(409 \mathrm{~ms})$. A significant main effect of SOA, $F_{(3,45)}=76.07, p<0.001$, partial $\eta^{2}=0.84$, indicated that RTs increased with decreasing SOA. This effect was more pronounced in the joint than in the individual condition (Figure 3A), as indicated by a significant interaction of condition $\times$ SOA, $F_{(3,45)}=52.25, p<0.001$, partial $\eta^{2}=0.78$. Planned $t$-tests (Bonferroni corrected to $\alpha^{\prime}<0.0125$ for four comparisons) showed that the difference in RTs between the joint and the individual condition was largest for the $0 \mathrm{~ms}$ SOA (238 ms, $p<0.001)$, followed by the $100 \mathrm{~ms}$ SOA $(170 \mathrm{~ms}$, $p<0.001)$, the $300 \mathrm{~ms} \mathrm{SOA}(79 \mathrm{~ms}, p<0.001)$, and the $1000 \mathrm{~ms}$ SOA (34 ms, $p<0.001)$.

Reaction time analysis for task 1. For Task 1, we observed a significant main effect of condition, $F_{(1,15)}=5.12, p<0.05$, partial $\eta^{2}=0.25$, indicating faster RTs in the joint condition $(360 \mathrm{~ms})$ as compared to the individual condition $(376 \mathrm{~ms})$. There was no significant main effect of SOA, $F<1$, partial $\eta^{2}=0.06$, and no significant interaction of condition $\times$ SOA, $F<1$, partial $\eta^{2}=$ 0.05 (Figure 3B).

\section{Transfer of task 1 motor priming on reaction times in task 2}

To test whether motor priming in Task 1 affects reaction times in Task 2, we calculated an ANOVA including the factors S1-R1 compatibility (compatible, incompatible) and $\operatorname{SOA}(0,100,300$, $1000 \mathrm{~ms}$ ), both as within-subject variables, separately for Task 2 and Task 1 of the joint condition, as well as the interaction of S1$\mathrm{R} 1$ compatibility and task for the $0 \mathrm{~ms}$ SOA.

Reaction time analysis for task 2. A significant main effect of S1-R1 compatibility, $F_{(1,15)}=8.01, p<0.05$, partial $\eta^{2}=0.35$, indicated faster RTs in Task 2 for compatible trials $(521 \mathrm{~ms})$ as compared to incompatible trials $(560 \mathrm{~ms})$. A significant main effect of SOA, $F_{(3,45)}=70.68, p<0.001$, partial $\eta^{2}=0.83$, indicated slower RTs for shorter SOAs than for longer SOAs. The size of the compatibility effect was larger for shorter SOAs $(59 \mathrm{~ms}$ for SOA $0 \mathrm{~ms}, p<0.025$, Bonferroni corrected to $\alpha^{\prime}<0.025$ for two comparisons) than for longer SOAs ( $8 \mathrm{~ms}$ for SOA $1000 \mathrm{~ms}$, $p>0.025)$, as indicated by a significant interaction of SOA $\times$ S1-R1 compatibility, $F_{(3,45)}=6.86, p<0.01$, partial $\eta^{2}=0.31$ (Figure 4A). 


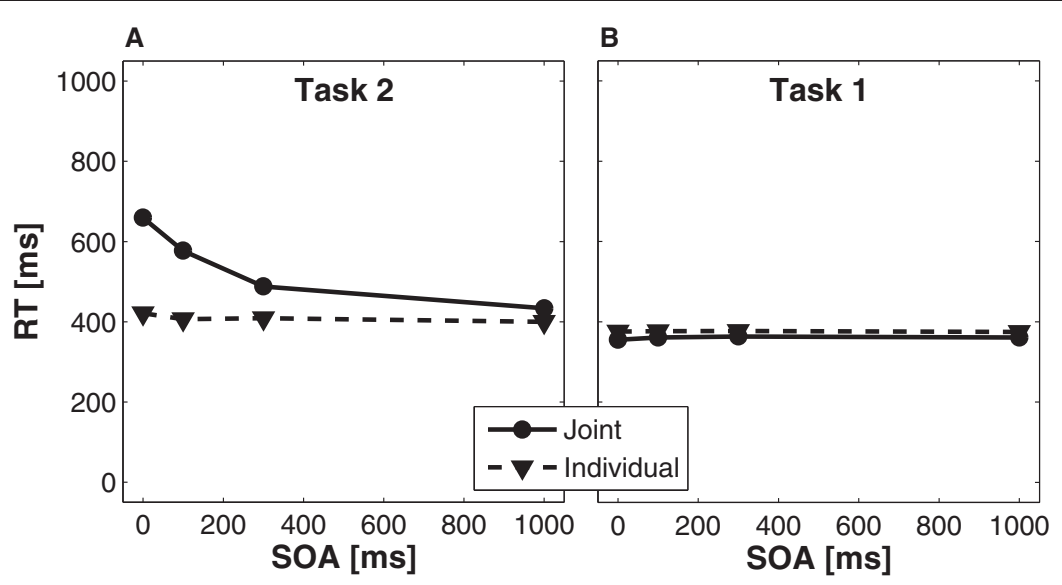

FIGURE 3 | Effects of stimulus onset asynchrony (SOA) on reaction times of Task 2 (A) and Task 1 (B), separately for the joint (solid lines) and individual (dashed lines) condition.

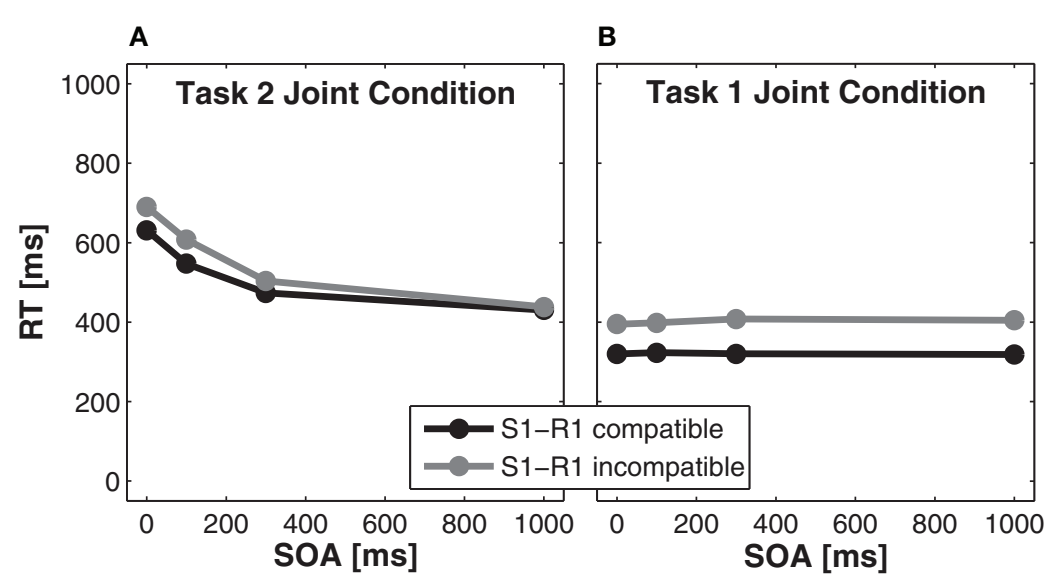

FIGURE 4 | Effects of stimulus onset asynchrony (SOA) on reaction times of Task 2 (A) and Task 1 (B) of the joint condition for $\mathrm{S}$ (timulus)1-R(esponse)1 compatible trials (black solid lines) and S1-R1 incompatible trials (gray solid lines).

Reaction time analysis for task 1 . A significant main effect of S1-R1 compatibility in task $1, F_{(1,15)}=66.76, p<0.001$, partial $\eta^{2}=0.82$, indicated that RTs were faster for compatible trials (321 ms) compared to incompatible trials $(402 \mathrm{~ms})$. A nonsignificant effect of SOA indicated that RTs did not differ for different SOAs, $F_{(3,45)}=1.41, p>0.05$, partial $\eta^{2}=0.09$. The interaction of SOA $\times$ S1-R1 compatibility was also not significant, $F_{(3,45)}=2.16, p>0.05$, partial $\eta^{2}=0.13$ (Figure 4B).

The interaction between S1-R1 compatibility $\times$ task was not significant, $F<1$, partial $\eta^{2}=0.02$, indicating a statistically comparable S1-R1 compatibility effect for Task $1(75 \mathrm{~ms})$ and Task 2 (59 ms) for the $0 \mathrm{~ms}$ SOA.

\section{TESTING TIME VERSUS CONTENT SPECIFIC ACTION EFFECT MONITORING}

Response content specific action effect monitoring

We analyzed RTs for Task 2 of the joint condition using an ANOVA including the factors R1-R2 compatibility (compatible, incompatible) and $\mathrm{SOA}(0,100,300,1000 \mathrm{~ms})$ to test if action effect monitoring depends on the correspondence of the specific finger responses jointly activated in both participants. A significant main effect of SOA, $F_{(3,45)}=68.98, p<0.001$, partial $\eta^{2}=$ 0.82 , indicated larger RTs for shorter SOAs. The main effect of R1-R2 compatibility was not significant, $F_{(1,15)}=2.16, p>0.05$, partial $\eta^{2}=0.13$, indicating that response-response crosstalk across both participants did not take place during joint task performance. We observed no significant interaction of SOA $\times$ R1-R2 compatibility, $F_{(3,45)}=2.77, p>0.05$, partial $\eta^{2}=0.16$ (Table 1).

\section{Monitoring of task rules}

To investigate whether the second person monitors the task rule of Person 1, we conducted an ANOVA including the factors S1-R2 compatibility (compatible, incompatible) and S1-R1 compatibility (compatible, incompatible), both as within-subjects variables, for RTs in Task 2 for the $0 \mathrm{~ms}$ SOA of the joint condition. We observed a significant main effect of S1-R1 compatibility, $F_{(1,15)}=8.29, p<0.05$, partial $\eta^{2}=0.36$, and a marginally 
Table 1 | Reaction times in milliseconds ( $\mathrm{ms}$ ) of Task 2 of the joint condition for $\mathbf{R}$ (esponse)1-R(esponse)2 compatible trials and R1-R2 incompatible trials for different stimulus onset asynchronies (SOAs).

\begin{tabular}{lll}
\hline & R1-R2 compatible & R1-R2 incompatible \\
\hline SOA 0 & 657 & 661 \\
SOA 100 & 586 & 568 \\
SOA 300 & 486 & 490 \\
SOA 1000 & 438 & 430
\end{tabular}

significant main effect of S1-R2 compatibility, $F_{(1,15)}=3.50, p<$ 0.10 , partial $\eta^{2}=0.19$ (note that only trials of the $0 \mathrm{~ms}$ SOA of the joint condition were included in the present analyses), indicating faster RTs for compatible trials than for incompatible trials. We observed no interaction of S1-R1 compatibility $\times$ S1-R2 compatibility, $F<1$, partial $\eta^{2}=0.03$, indicating that the size of S1-R2 compatibility effect was not modulated by S1-R1 compatibility. The S1-R2 compatibility effect for S1-R1 compatible trials amounted to $22 \mathrm{~ms}$ and the S1-R2 compatibility effect for S1-R1 incompatible trials amounted to $12 \mathrm{~ms}$.

\section{DISCUSSION}

The present study combined an automatic imitation task and a social dual-task paradigm to test if automatic imitation effects break down during joint action. Further, we aimed to test if task monitoring is time or content dependent.

In line with previous studies from action observation (CalvoMerino et al., 2005) and imitation research (Brass et al., 2000; Iacoboni et al., 2005), we observed a motor priming effect based on the correspondence between the observed and executed actions for Person 1 and for Person 2 in the individual condition. Extending these findings, we also observed motor priming effects for both persons during active engagement in joint action. These findings suggest that direct matching is not overridden by the active engagement of two persons during joint dual-task processing.

Further, we observed an increase of reaction times with decreasing SOA for the joint condition replicating the finding of a social PRP effect with realistic hand stimuli. The social PRP effect seems, therefore, not to be restricted to fully overlapping task sets and abstract number categorization tasks, as present in Liepelt and Prinz (2011). For the zero SOA condition, we further observed a full transfer of the S1-R1 compatibility effect to the reaction times of Person 2. As the task setting we used forced Person 2 to supervise the action effects produced by Person 1 and the delay of reaction times in Person's 1 task was based on a manipulation of the response selection stage in Task 1, we assume that the transfer effect found for Person 2 is due to strategic action effect monitoring.

As we did not find evidence for response-response crosstalk across both persons during joint task processing, action effect monitoring seems not to be related to the specific response given by the other person. Further, motor priming for Person 2 was not affected by the way Person 1 responded to the hand stimulus (imitation vs. counter-imitation). This suggests that the present social dual-task situation may also not require the co-representation of the other's specific S-R mappings (Sebanz et al., 2005). Taken together, our findings suggest that the action effect monitoring process we observed is related to the point in time when the other person responds and may not be related to the specific task-content.

The presence or expectation (Vlainic et al., 2010) of another responding person represents a salient event that provides an alternative for the actor's own response (Guagnano et al., 2010; Dolk et al., 2011). As Person 1 acts ahead in time, a slight head start may even increase the saliency of the response given by this person. One may speculate that the difficulty to distinguish the cognitive representation of one's own action from the representation of the others action (Dolk et al., 2011; Liepelt et al., in press) increases, the more the time interval between both stimuli decreases rendering the effects of joint task performance particularly strong at the shorter SOAs. Person 2 may run some inner time clock, monitoring the production of the first person's action effects.

Action effect monitoring may also be relevant in real life. For example, when two football players supervise the other person's action effects before starting their own run in order to properly achieve joint goal states. What is needed for a successful goal achievement is an effective resource allocation process in space and time (Vygotsky, 1978; Meyer and Kieras, 1997b) that allows smooth inter-personal action (Sebanz et al., 2006) and inter-task coordination (Liepelt et al., 2011b). While the present findings tap the process of allocation of resources with respect to time, other studies investigating joint action within the spatial domain, such as the Social Simon effect (Sebanz et al., 2003; Liepelt et al., 2011c), may tap the allocation of resources in space.

A possible concern regarding the present findings is that a monitoring of the exact S-R mappings of the other co-actor is not needed to succeed in joint task performance in our study. The effects of content specific task monitoring may, therefore, only be found in the present task situation when task monitoring would be a fully automatic process, which seems not to be the case. A task situation that would force both participants to activate the other person's S-R mappings, as for example by relating the response of Person 2 more specifically to the task-content of Person 1, may, therefore, provide evidence for content specific task monitoring effects. We think that this would be an interesting way to go in future research since such a social setting seems to occur quite often in real life.

\section{CONCLUSION}

Taken together, our findings suggest that direct matching is not disturbed during joint action. Action effect monitoring in the present version of the social dual-task seems to be time dependent. The assumption that we represent the exact S-R mappings of the co-actor is not necessary to explain the observed social PRP effect.

\section{ACKNOWLEDGMENTS}

We would like to thank Eva Güthling and Felix Rebitschek for help with data acquisition, Marcel Brass for providing the automatic imitation stimulus and two reviewers for helpful comments on a previous version of this article. This research was supported by the German Research Foundation project DFG LI 2115/1-1. 


\section{REFERENCES}

Beringer, J. (2000). Experimental Runtime System. Frankfurt am Main (1987-2000): BeriSoft Cooperation.

Bertenthal, B. I., Longo, M. R., and Kosobud, A. (2006). Imitative response tendencies following observation of intransitive actions. J. Exp. Psychol. Hum. Percept. Perform. 32, 210-225.

Brass, M., Bekkering, H., Wohlschläger, A., and Prinz, W. (2000). Compatibility between observed and executed finger movements: comparing symbolic, spatial, and imitative cues. Brain Cogn. 44, 124-143.

Brass, M., and Heyes, C. M. (2005). Imitation: is cognitive neuroscience solving the correspondence problem? Trends Cogn. Sci. 9, 489-495.

Buccino, G., Binkofski, F., Fink, G. R., Fadiga, L., Fogassi, L., Gallese, V., Seitz, R. J., Zilles, K., Rizzolatti, G., and Freund, H. J. (2001). Action observation activates premotor and parietal areas in a somatotopic manner: an fMRI study. Eur. J. Neurosci. 13, 400-404.

Calvo-Merino, B., Glaser, D., Grezes, J., Passingham, R., and Haggard, P. (2005). Action observation and acquired motor skills: an fMRI study with expert dancers. Cereb. Cortex 15, 1243-1249.

Dolk, T., Hommel, B., Colzato, L. S., Schütz-Bosbach, S., Prinz, W., and Liepelt, R. (2011). How social is the social Simon effect? Front. Psychol. 2:84. doi: 10.3389/fpsyg.2011.00084

Fogassi, L., Ferrari, P. F., Gesierich, B., Rozzi, S., Chersi, F., and Rizzolatti, G. (2005). Parietal lobe: from action organization to intention understanding. Science 308, 662-667.

Gallese, V., Fadiga, L., Fogassi, L., and Rizzolatti, G. (1996). Action recognition in the premotor cortex. Brain $119,593-609$.

Gallese, V., and Goldman, A. (1998). Mirror neurons and the simulation theory of mind-reading. Trends Cogn. Sci. 12, 493-501.

Graf, M., Reitzner, B., Corves, C., Casile, A., Giese, M., and Prinz, W. (2007). Predicting point-light actions in real-time. Neuroimage 36, T22-T32.

Guagnano, D., Rusconi, E., and Umiltà, C. A. (2010). Sharing a task or sharing space? On the effect of the confederate in action coding in a detection task. Cognition 114, 348-355.

Hommel, B. (1998). Automatic stimulus-response translation in dual-task performance. J. Exp.
Psychol. Hum. Percept. Perform. 24, 1368-1384.

Iacoboni, M., Molnar-Szakacs, I., Gallese, V., Buccino, G., Mazziotta, J. C., and Rizzolatti, G. (2005). Grasping the intentions of others with one's own mirror neuron system. PLoS Biol. 3:e79. doi: 10.1371/journal.pbio.0030079

Iacoboni, M., Woods, R. P., Brass, M., Bekkering, H., Mazziotta, J. C., and Rizzolatti, G. (1999). Cortical mechanisms of human imitation. Science 286, 2526-2528.

Lien, M.-C., and Proctor, R. W. (2000). Multiple spatial correspondence effects on dual-task performance. J. Exp. Psychol. Hum. Percept. Perform. 26, 1260-1280.

Liepelt, R., and Brass, M. (2010a). Automatic imitation of physically impossible movements. Soc. Cogn. 28, 59-74.

Liepelt, R., and Brass, M. (2010b). Topdown modulation of motor priming by belief about animacy. Exp. Psychol. 57, 221-227.

Liepelt, R., Fischer, R., Frensch, P., and Schubert, T. (2011a). Practice-related reduction of dualtask costs under conditions of a manual-pedal response combination. J. Cogn. Psychol. 23, 29-44.

Liepelt, R., and Prinz, W. (2011). How two share two tasks: evidence of a social psychological refractory period effect. Exp. Brain Res. 211, 387-396.

Liepelt, R., Prinz, W., and Brass, M. (2010). When do we simulate non-human agents? Dissociating communicative and non-communicative actions. Cognition 115, 426-434.

Liepelt, R., Strobach, T., Frensch, P., and Schubert, T. (2011b). Improved Inter-task Coordination Skills after extensive Dual-task Practice. Q. J. Exp. Psychol. 64, 1251-1272.

Liepelt, R., von Cramon, D. Y., and Brass, M. (2008a). What is matched in direct matching? Intention attribution modulates motor priming. J. Exp. Psychol. Hum. Percept. Perform. 34, 578-591.

Liepelt, R., von Cramon, D. Y., and Brass, M. (2008b). How do we infer others' goals from non-stereotypic actions? The outcome of contextsensitive inferential processing in right inferior parietal and posterior temporal cortex. Neuroimage $43,784-792$.
Liepelt, R., Wenke, D., and Fischer, R. (in press). Effects of Feature Integration in a Hands-Crossed Version of the Social Simon Paradigm. Psychol. Res. doi: 10.1007/s00426-012-0425-0

Liepelt, R., Wenke, D., Fischer, R, and Prinz, W. (2011c). Trial-to-trial sequential dependencies in a social and non-social Simon task. Psychol. Res. 75, 366-375.

Meyer, D. E., and Kieras, D. E. (1997a). A computational theory of executive cognitive processes and multipletask performance: part 1. Basic mechanisms. Psychol. Rev. 104 3-65.

Meyer, D. E., and Kieras, D. E. (1997b). A computational theory of executive cognitive processes and multipletask performance: part 2. Account of psychological refractory-period phenomena. Psychol. Rev. 104 749-791.

Pashler, H. (1984). Processing stages in overlapping tasks: evidence for a central bottleneck. J. Exp. Psychol. Hum. Percept. Perform.10 358-377.

Pashler, H. (1994). Dual-task interference in simple tasks: data and theory. Psychol. Bull. 116, 220-244.

Pashler, H., and Johnston, J. C. (1989). Chronometric evidence for central postponement in temporally overlapping tasks. Q. J. Exp. Psychol. $41 \mathrm{~A}, 19-45$.

Schubert, T., Fischer, R., and Stelzel, C. (2008). Response activation and the central bottleneck in overlapping tasks. J. Exp. Psychol. Hum Percept. Perform. 34, 376-397.

Schubotz, R. I. (2007). Prediction of external events with our motor system: towards a new framework. Trends Cogn. Sci. 11, 211-218.

Schumacher, E. H., Seymour, T. L., Glass, J., Lauber, E. H., Kieras, D. E. and Meyer, D. E. (2001). Virtually perfect time-sharing in dual-task performance: uncorking the central cognitive bottleneck. Psychol. Sci. 121, 101-108.

Sebanz, N., Bekkering, H., and Knoblich, G. (2006). Joint action: bodies and minds moving together. Trends Cogn. Sci. 10, 70-76.

Sebanz, N., and Knoblich, G. (2009). Prediction in joint action: what, when, and where. Top. Cogn. Sci. 1, 353-367.

Sebanz, N., Knoblich, G., and Prinz, W. (2003). Representing others' actions: just like one's own? Cognition 88, B11-B21.
Sebanz, N., Knoblich, G., and Prinz, W. (2005). How two share a task: corepresenting stimulus-response mappings. J. Exp. Psychol. Hum. Percept. Perform. 31, 1234-1246.

Springer, A., Brandstädter, S., Liepelt, R., Birngruber, T., Giese, M., Mechsner, F., and Prinz, W. (2011). Motor execution affects action prediction. Brain Cogn. 76, 26-36.

Stadler, W., Schubotz, R. I., von Cramon, D. Y., Springer, A., Graf, M., and Prinz, W. (2011). Predicting and memorizing observed action: differential premotor cortex involvement. Hum. Brain Mapp. 32, 677-687.

Telford, C. W. (1931). The refractory phase of voluntary and associative responses. J. Exp. Psychol. 14, $1-36$.

Tombu, M., and Jolicoeur, P. (2004). Virtually no evidence for virtually perfect time-sharing. J. Exp. Psychol. Hum. Percept. Perform. 30, 795-810.

Vlainic, E., Liepelt, R., Colzato, L. S., Prinz, W., and Hommel, B. (2010). The virtual co-actor: the Social Simon effect does not rely on online feedback from the other. Front. Psychol. 1:208. doi: 10.3389/fpsyg.2010.00208

Vygotsky, L. S. (1978). Mind in Society: The Development of Higher Psychological Processes. Cambridge, MA: Harvard Business Press.

Welford, A. T. (1952). The "psychological refractory period" and the timing of high speed performance. Br. J. Psychol. 43, 2-19.

Conflict of Interest Statement: The authors declare that the research was conducted in the absence of any commercial or financial relationships that could be construed as a potential conflict of interest.

Received: 23 December 2011; accepted: 26 March 2012; published online: 18 April 2012

Citation: Liepelt $R$, Stenzel $A$ and Lappe $M$ (2012) Specifying social cognitive processes with a social dual-task paradigm. Front. Hum. Neurosci. 6:86. doi: 10.3389/fnhum.2012.00086

Copyright (๑) 2012 Liepelt, Stenzel and Lappe. This is an open-access article distributed under the terms of the Creative Commons Attribution Non Commercial License, which permits non-commercial use, distribution, and reproduction in other forums, provided the original authors and source are credited. 